\title{
MARCO ANALITICO PARA EL APROVECHAMIENTO DE TECNOLOGIAS DE INFORMACIÓN Y COMUNICACIÓN. DIAGNÓSTICO EN ORGANIZACIONES GUBERNAMENTALES Y DE LA SOCIEDAD CIVIL EN MÉXICO
}

\section{ANALYTICAL FRAMEWORK FOR THE USE OF INFORMATION AND COMMUNICATION TECHNOLOGIES. DIAGNOSIS IN GOVERNMENT AND CIVIL SOCIETY ORGANIZATIONS IN MEXICO}

\author{
María Rebeca Padilla de la Torre \\ Univ. Autónoma de Aguascalientes, México \\ http://orcid.org/0000-0002-5881-3958 \\ rebeca.padilla@edu.uaa.mx
}

\author{
Ana Isabel Zermeño Flores \\ Universidad de Colima, México \\ http://orcid.org/0000-0001-7371-8767 \\ anaz@uco.mx
}

\author{
Thomas Tufte \\ Loughborough University, London, United Kingdom \\ http://orcid.org/0000-0003-3253-8481 \\ T.Tufte@lboro.ac.uk
}

Para citar este artículo:

Padilla de la Torre, M.R., Zermeño Flores, A.I., \& Tufte, T. (2019). Marco analítico para el aprovechamiento de Tecnologías de Información y Comunicación. Diagnóstico en organizaciones gubernamentales y de la sociedad civil en México. Commons. Revista de Comunicación y Ciudadanía Digital, 8(1), 44-75.

http://dx.doi.org/10.25267/COMMON.2019.v8.i1.02

Fecha de recepción: 09/03/2019. Fecha de aceptación: 26/04/2019

\section{Resumen}

Este artículo tiene como objetivo proponer un marco analítico para el aprovechamiento de las TIC en organizaciones gubernamentales y de la sociedad civil con fines sociales, el cual se integró a través de estudios y encuestas previas. Este marco sirvió de base para el análisis de los datos derivados de una encuesta en línea aplicada a 203 organizaciones mexicanas centradas en el desarrollo de los jóvenes. Los hallazgos muestran el nivel de aprovechamiento de las TIC con relación a tres niveles de uso (básico, intermedio y alto) y 11 dimensiones. Se encuentra que los usos de las TIC se aprovechan principalmente con fines administrativos y de difusión y en menor medida para potenciar sus capacidades para responder a las problemáticas sociales que atienden. El estudio genera conocimiento sobre organizaciones que son agentes claves para el desarrollo y el cambio social y es una propuesta replicable para otras organizaciones y contextos.

\section{Palabras clave}

Tecnologías de información y comunicación, comunicación y desarrollo, organizaciones gubernamentales, organizaciones de la sociedad civil, jóvenes

\section{Abstract}

The objective of this paper is to propose an analytical framework for the use of ICT in governmental and civil society organizations for social development, which was integrated through the review of previous studies and surveys. Data from an online survey applied to 203 mexican organizations focused on the development of young people were analyzed based on this framework. The findings show the level of use of ICT in relation to three levels of use (basic, intermediate and high) and 11 dimensions. It is found that the use of ICTs is mainly for administrative and dissemination purposes and to a lesser extent to enhance their capacities to respond to the social problems they address. This study generates knowledge about these organizations which are key actors for development and social change. Addtionally, this proposal is replicable in other organizations and contexts.

\section{Keywords}

Information and communication technologies, communication and development, governmental organization, non-governmental organization, youth 


\section{Introducción}

El desarrollo social, en el caso de México, es un asunto apremiante debido a que es un país que histórica y estructuralmente ha presentado graves desigualdades de distinta naturaleza como la económica, de género y territorial, entre otras, que influyen en el bienestar de sus ciudadanos. A pesar de que el más reciente estudio económico de la Organización para la Cooperación y el Desarrollo Económicos (OCDE, 2018) señala que México ha mejorado su crecimiento económico, su resiliencia y se ha situado como la undécima economía del mundo, las desigualdades y vulnerabilidades entre su población son las más altas entre los países que integran la OCDE. El estudio de las desigualdades es una perspectiva más amplia con respecto al análisis de los niveles de pobreza de un país, las cuales se interrelacionan y acumulan, y tienen un grave impacto no solo con respecto a la carencia de recursos y falta de oportunidades a nivel individual, sino en la calidad de la convivencia, solidaridad y participación política de una comunidad. Las desigualdades se comprenden como "las distribuciones inequitativas de resultados y acceso a oportunidades entre individuos o grupos" (El Colegio de México, 2018: 20).

Los distintos aspectos de las desigualdades entre la población han sido atendidos por organizaciones de gobierno (OG) y de la sociedad civil (OSC) y la naturaleza de estas son muy diversas, trabajan dentro de situaciones muy complejas e incluso su papel ha sido cuestionado. No obstante, han sido y son estratégicas para promover el desarrollo de la población vulnerable y marginada (Reygadas, 2005). Por ello, es necesario fortalecer su capacidad para lograr su misión social, y en el escenario contemporáneo, las tecnologías de la información y la comunicación (TIC) son un elemento clave para alcanzar este propósito. 
El objetivo de este artículo es ofrecer un marco analítico para el diagnóstico sobre los usos y aprovechamiento de las TIC en OG y OSC ${ }^{1}$. Esta propuesta teórica permitió el análisis de los datos de una encuesta aplicada a finales del 2016 a 203 organizaciones mexicanas cuyo propósito fuera el desarrollo de los jóvenes. Este marco se construyó con base en las tendencias internacionales para el aprovechamiento de las TIC en organizaciones con una misión social. A partir de dicha revisión se construyó una clasificación con tres niveles de usos de las TIC (básico, intermedio y alto) que integran, a su vez, 11 dimensiones que dan cuenta del alcance y aprovechamiento de las TIC en el marco de su quehacer social. La clasificación trasciende el nivel técnico-instrumental de la relación con las TIC y valora su adopción para el desarrollo en un horizonte que va desde su propia capacidad organizacional hasta sus posibilidades para insertarse en procesos de desarrollo y cambio social globales.

El artículo aborda, en primer lugar, un marco teórico y referencial sobre desarrollo, organizaciones y jóvenes, y un panorama general sobre la situación de las OG y OSC en México que se centran en la problemática de los jóvenes en este país; así como el papel de las TIC en organizaciones con un propósito social. Posteriormente se da a conocer la metodología de la aplicación de la encuesta nacional a OG y OSC. Después, se presentan los resultados sobre la capacidad organizacional, la infraestructura y acceso a TIC y los niveles de aprovechamiento de las organizaciones. Finalmente, se discuten los hallazgos y se brindan las conclusiones que revelan la realidad en la que están las organizaciones con respecto al horizonte de posibilidades que abren las TIC para el desarrollo social.

1. Esta encuesta formó parte de un estudio más amplio: “Tecnologías de la Información y Comunicación en Organizaciones Gubernamentales y Civiles para el Desarrollo de los Jóvenes”. El proyecto contempló una metodología mixta, la aproximación cuantitativa fue a través de esta encuesta y la cualitativa consistió en trabajar en seis casos de organizaciones en distintas ciudades del país para analizar cómo incorporar las TIC de manera significativa a su quehacer. Los resultados de esta segunda fase no se presentan en este artículo. Para mayor información sobre esta investigación se pueden consultar en el sitio web www.itinerariosparaorganizaciones.com 


\section{Marco teórico y contextual}

Este estudio se sitúa en el campo de la Comunicación para el Desarrollo y el Cambio Social (Tufte, 2015) y más específicamente en la perspectiva de las TIC para el Desarrollo, ICT4D (por sus siglas en inglés) (Kleine, 2013; Walsham, 2017). En esta última perspectiva se define el concepto de desarrollo a partir de la teoría de las capacidades de Sen (2000). Además, dado que el diagnóstico se centró en organizaciones orientadas hacia los jóvenes, se asume el enfoque de los estudios e intervenciones para el desarrollo juvenil que promueve una comprensión integral del potencial de los jóvenes en lugar de focalizarse en un único comportamiento problemático (Catalano, Berglund, Ryan, Lonczak \& Hawkins, 2004).

\subsection{Las organizaciones y el desarrollo social en México}

Por organización social "nos referimos a organizaciones cuyos objetivos son atender problemáticas sociales, contribuir al bienestar, transmitir valores y reflexionar acerca de la sociedad. Tal es la lógica del sector social" (Enz, Franco \& Spagnuolo, 2012: 21). Este concepto puede aplicarse para el estudio tanto de OG como OSC que atienden problemáticas juveniles. Cabe destacar que, a las OSC, también se les conoce como organizaciones no gubernamentales (ONG) u organizaciones sin fines de lucro (OSFL).

Las OG y OSC son estratégicas para la política social de los países. Lizarraga (2004) distingue que, hasta antes de los años 80 del siglo pasado, la relación entre estas organizaciones era mayoritariamente de desconfianza y antagonismo y solía verse a las OSC desde una perspectiva idealista mientras que a las OG desde el prejuicio y la sospecha. La autora identifica algunas condiciones que significaron un punto de inflexión en las formas de ver y atender los problemas sociales y que favorecieron la colaboración entre OG y OSC. Estas condiciones fueron el cambio en los lineamientos internacionales sobre desarrollo, el auge de la participación de la sociedad civil y la agudización o surgimiento de problemas a nivel global de tipo ecológico, supervivencia y salud. A esta lista puede agregarse la inclusión digital y la atención a grupos sociales específicos en condición vulnerable como mujeres, indígenas, personas de la tercera edad y jóvenes. 


\subsection{Organizaciones centradas en el desarrollo de los jóvenes}

Tradicionalmente, las instituciones responsables de atender a los jóvenes han sido la familia y la escuela, e indudablemente con mayor peso y relevancia. Sin embargo, en este planteamiento se visibiliza el papel de las organizaciones como un sector alterno y no formal que apoya a los jóvenes ante diferentes problemáticas.

Varios estudios sobre jóvenes (Durand, 2017; Negrete \& Leyva, 2013; Reguillo, 2012) identifican que aun cuando hay una mejora en el estado de bienestar en general y en particular respecto a los jóvenes de generaciones anteriores, este grupo de población sigue en riesgo estructural debido a la desescolarización y a la baja calidad educativa, escasez de oportunidades para acceder a empleos de calidad que les permitan independencia económica y sentido de realización profesional, falta de acceso al sistema de salud versus la preeminencia de embarazos tempranos, adicciones y muertes por violencia. Estas organizaciones o colectivos generalmente se sitúan en problemáticas concretas, como lo son el disminuir el retraso educativo, habilitar para el trabajo, atender problemas de salud, familiares, delictivos o incluso de identidad y culturales, a partir de varias estrategias de trabajo social y comunitario. 
2.3. Uso de TIC en organizaciones para el desarrollo social y de los jóvenes

En las pasadas cumbres internacionales de la sociedad de la información (World Summit on the Information Society, WSIS, 2018) se ha argumentado que las TIC permiten a las organizaciones un mejor acceso a la información; a la administración y generación del conocimiento; a visibilizar y movilizar apoyos para sus causas; a fortalecer su capacidad organizacional; así como promover el empleo sostenible de las TIC entre las comunidades y sectores de la población que atienden. Además, la ONU (2010) resalta que los procesos de apropiación de las TIC no deben ser aislados, estos esfuerzos deben integrarse de manera global para optimizar las lecciones aprendidas, las estrategias implementadas por las políticas públicas, los actores sociales locales e internacionales, así como los sistemas de evaluación de resultados que se han propuesto. Coinciden en esta postura Finquelievich y Kisilevsky (2005) quienes analizaron el papel de las TIC en OSC en Argentina y concluyen que la apropiación de estas debe trascender su uso en procesos administrativos, difusión de la imagen y consecución de apoyos (voluntarios y financiamiento) para aspirar a un mayor alcance como catalizadoras del cambio social. A su vez, Camacho (2003) identificó cómo se han empleado en las organizaciones las TIC con éxito en procesos de transformación. Destaca varias funciones que van desde la mejor gestión de la organización interna de estas instancias hasta un empleo de las TIC que trasciende las organizaciones para generar conocimiento y promover posicionamientos para el desarrollo social.

Sin embargo, De Silva (2016) afirma que, a pesar de que la mayoría de las organizaciones emplean TIC, no siempre logran aprovecharlas de manera óptima y se requieren mayores esfuerzos para garantizar su uso efectivo de acuerdo a su misión social. Otro aspecto clave para su mejor aprovechamiento es la identificación del campo problemático donde se aplican. Walsham (2017) argumenta que se trata de un reto ético el indagar el valor de las TIC para contribuir a la diversidad de problemas actuales. Los esfuerzos de los estudios e intervenciones de ICT $4 D$ se han centrado predominantemente en incorporar equipamiento y formar habilidades digitales en comunidades marginadas y vulnerables, con menos trabajo especializado en el nivel organizacional que permite impulsar el desarrollo desde otra perspectiva. 
El análisis del uso estratégico de las TIC en organizaciones va más allá de revisar solo su infraestructura y acceso para indagar en el aprovechamiento y el alcance que hacen de estas tecnologías. Enseguida se describe la aproximación metodológica que se siguió para realizar un diagnóstico entre organizaciones mexicanas.

\section{Estrategia metodológica. Encuesta nacional a OG y OSC en México}

La metodología consistió en una encuesta en línea con seguimiento y acompañamiento telefónico realizada en el segundo semestre del 2016 a 203 OG (95) y OSC (108) a nivel nacional que atendieran problemáticas relacionadas con los jóvenes de 12 a 29 años en México.

\section{1. Diseño y validación del instrumento}

Al momento de realizar el diseño del instrumento, se identificaron los siguientes diagnósticos y estudios internacionales sobre usos de TIC en organizaciones (Attouni \& Mustaffa, 2014; Nugroho, 2011; Finquelievich \& Kisilevsky, 2005; Camacho, 2003), así como algunas encuestas nacionales que incluían un apartado sobre TIC (INEGI, 2017; INEI, 2007). La estructura del instrumento de recolección de información fue la siguiente (Tabla I): 
Tabla I

Estructura del instrumento de recolección

\begin{tabular}{|l|l|c|}
\hline Sección & \multicolumn{1}{|c|}{ Temas } & Número de ítems \\
\hline I & $\begin{array}{l}\text { Datos generales de la organización/ } \\
\text { institución }\end{array}$ & $1-10(10)$ \\
\hline II & $\begin{array}{l}\text { Objetivos, población atendida y estrategias } \\
\text { de intervención }\end{array}$ & $11-22(12)$ \\
\hline III & $\begin{array}{l}\text { Uso de tecnologías de la información y la } \\
\text { comunicación (TIC) }\end{array}$ & $23-42(20)$ \\
\hline IV & $\begin{array}{l}\text { Estructura organizacional, infraestructura } \\
\text { física y financiamiento }\end{array}$ & $43-49(7)$ \\
\hline & \multicolumn{2}{|c|}{ Total } \\
\hline
\end{tabular}

Fuente: elaboración propia

La validación teórica conceptual y pertinencia de los ítems del instrumento se realizó a través de la discusión y juicio por parte del equipo de investigación "Agorante" ${ }^{2}$ de la Universidad de Colima en México, especializado en tecnologías para el desarrollo. Este grupo ha liderado proyectos a nivel nacional sobre inclusión digital para el gobierno federal a través de la Coordinación de la Sociedad de la Información y el Conocimiento (CSIC) de la Secretaría de Comunicaciones y Transportes (SCT), órgano del estado que encabeza la Agenda Digital en el país. Cabe señalar que uno de esos proyectos obtuvo el primer lugar en la dimensión ética de la Sociedad de la Información, Premio WSIS 2013, que otorga la Unión Internacional de Telecomunicaciones (UIT) de la Organización de las Naciones Unidas (ONU). Mientras que la validación semántica de los ítems se realizó a través de una prueba piloto vía telefónica a cuatro organizaciones y se realizaron los ajustes correspondientes, la consistencia interna del cuestionario se ejecutó a través de la prueba de confiabilidad de Cronbach.

2. Mayor información sobre este equipo de investigación puede consultarse en: www.agorante.mx 
En la sección tres del instrumento, que recoge información sobre los usos de TIC, se realizaron diversos análisis de confiabilidad de la consistencia dado que este apartado se refiere al objetivo central del estudio. En todos los casos, se obtuvo un alfa de Cronbach de entre 0.74 y 0.86 , lo que indica un nivel aceptable y bueno de la confiabilidad interna de este instrumento.

\subsection{Construcción de la muestra}

Para definir el universo muestral se construyó un directorio en el cual se identificaron 233 OG y 643 OSC que atendieran problemáticas relacionadas con los jóvenes en México. La estrategia para identificar las OG consistió en revisar los sitios web de los 32 gobiernos estatales y sus municipios, en los cuales se daban a conocer sus instancias responsables de programas sociales dirigidos a jóvenes. En el caso de las OSC, se identificaron las registradas en el Sistema de Información del Registro Federal de las OSC (SIRFOSC) del Instituto Nacional de Desarrollo Social en México que incluyeran en su denominación las palabras "juvenil", "jóvenes", "adolescentes" o "juventud".

Debe notarse que este directorio es muy efímero en el tiempo. Nuestra experiencia en campo nos indica que esto se debe a que las OG se crean y desaparecen en México en función de los programas sociales gubernamentales sexenales. Por otra parte, las OSC incluyen una miríada de organizaciones formales e informales, de tamaño mínimo (una persona) hasta un colectivo; y operan con recursos gubernamentales y en menor medida con recursos propios ${ }^{3}$. En este sentido, el directorio formado en el marco de esta investigación es un indicativo para definir una muestra estadística.

3. Es importante señalar que "organizaciones de la sociedad civil" es un término amplio que contempla el ámbito en que los ciudadanos, los movimientos sociales o populares se organizan en torno a determinados objetivos, grupos de personas o temas de interés; también se denominan organizaciones no gubernamentales o del tercer sector. Por su parte, las asociaciones civiles son organizaciones de carácter privado que disponen de personalidad jurídica y que tienen fines no lucrativos para trabajar a favor de propósitos sociales, educativos, culturales o de otro tipo. 
El esquema de muestreo se realizó a partir de una pre-muestra (10 encuestas por tipo de organización, esto es gubernamental o de la sociedad civil), donde se toman los años de operación de las instituciones como variable de análisis. Los años de operación expresan la capacidad de gestión y pertinencia social que tiene una organización gubernamental o la sociedad civil en su labor cotidiana. La fórmula empleada y el procedimiento se basan en Montesinos-López et al. (2019) (Imagen 1).

\section{Imagen 1}

Fórmula para muestreo aleatorio

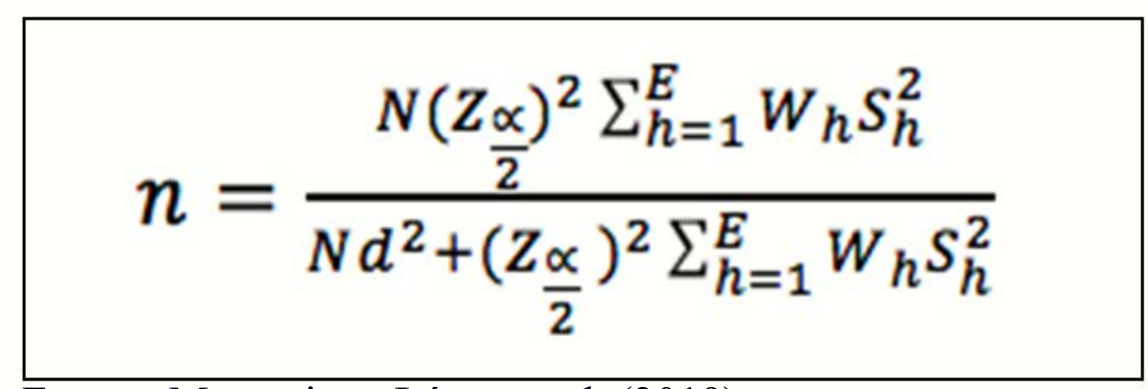

Fuente: Montesinos-López et al. (2019)

Donde: $\mathrm{N}$ (población) $=876 ;=(1.96)^{2}=3.841 ; \mathrm{d}=$ (precisión*promedio estratificado) $=0.0866 * 14.1=1.2227 ;=103.27$ (véase Tabla V). Con estos datos se tiene un tamaño de muestra de $n=203$ OG u OSC a encuestar. 
Tabla II

Información de la pre-muestra

\begin{tabular}{|c|c|c|c|c|c|c|c|}
\hline $\begin{array}{c}\text { Tipo de } \\
\text { organización / } \\
\text { institución }\end{array}$ & $\begin{array}{c}\text { Tamaño } \\
\text { del } \\
\text { Estrato } \\
\text { ( ) }\end{array}$ & $\begin{array}{c}\text { Varianza* } \\
=\mathbf{~ ( ~ ) ~}\end{array}$ & /N=( ) & ( ) & Afijación & Aplicadas & $\begin{array}{c}\text { Útiles } \\
\text { para el } \\
\text { análisis }\end{array}$ \\
\hline $\begin{array}{c}\text { Gubernamental } \\
233\end{array}$ & 150.44 & 0.266 & 40.015 & 54 & 124 & 95 \\
\hline $\begin{array}{c}\text { De la sociedad } \\
\text { civil }\end{array}$ & 643 & 86.18 & 0.734 & 63.256 & 149 & 134 & 108 \\
\hline SUMA & 876 & & & 103.272 & 203 & 258 & 203 \\
\hline
\end{tabular}

Fuente: elaboración propia ${ }^{4}$

La meta de encuestar OG y OSC se logró al aplicar un seguimiento telefónico, identificando informantes clave, durante el periodo de julio a octubre del 2016. Tratar de alcanzar el tamaño de muestra preestablecido, en particular del tipo OSC, fue particularmente difícil por el problema de la informalidad de muchas organizaciones. En total se aplicaron 258 encuestas, pero no todos los representantes que respondieron al cuestionario lo hicieron con el compromiso necesario en lo relativo a la consistencia de sus respuestas o en responder a todo el cuestionario; por lo que finalmente resultaron 95 encuestas a OG y 108 a OSC como útiles para fines de análisis estadístico (Tabla II).

4. */ Son valores para una prueba piloto de 10 encuestas para cada tipo de organización, esto es 20 encuestas. 


\section{Resultados}

Los resultados se discuten en dos apartados: el primero describe el perfil de las organizaciones en tanto actores para el cambio social, es decir, observa sus capacidades para responder a los desafíos de su misión; el segundo revisa los usos de TIC con relación al horizonte ideal de su aprovechamiento para el desarrollo.

\subsection{Perfiles y capacidad organizacional de OG y OSC}

Palos y Méndez (2011) señalan que existen diversas figuras legales entre las organizaciones que tienen una misión social, los resultados de este estudio coinciden al identificar varios tipos de estas que atienden a los jóvenes en México. Entre las OSC predominan las asociaciones civiles (92.6\%), frente a un equilibrio minoritario de sociedades civiles (3.7\%) e instituciones de asistencia privada (3.7\%); mientras que las OG se distinguen de acuerdo al nivel de gobierno, sea federal, estatal o municipal, además de existir organismos públicos descentralizados. En México, los organismos descentralizados son organizaciones creadas por la Ley del Congreso de la Unión o por el presidente de México. La descentralización consiste en trasladar ciertos servicios o funciones que desarrollaba un poder (ejecutivo, legislativo o judicial) hacia estas instituciones que serán autónomas y contarán con su propio presupuesto.

Entre las OG destaca el papel de las municipales, dado que el $54.7 \%$ de las organizaciones que contestaron a la encuesta corresponden a este nivel; le siguieron los organismos descentralizados con el $27.4 \%$, las dependencias del gobierno estatal con $16.8 \%$ y las federales con una mínima presencia del 1.1\%. 
En México, la principal organización de gobierno dedicada a los jóvenes es el Instituto Mexicano de la Juventud (IMJUVE) creado en 1999 y cada estado cuenta con una instancia estatal, que puede ser una Comisión, Instituto o Secretaría. Además, existen áreas de operación en la mayoría de los 2.446 municipios y las 16 delegaciones de la Ciudad de México.

Las organizaciones con mayor antigüedad son las de la sociedad civil, la más antigua informó que comenzó en 1922, mientras que en el caso de las OG la primera se reporta en 1970 . Además, poco más de un tercio (31.5\%) de las OSC fueron creadas durante o antes del año 2000, mientras que un porcentaje menor, el $15.8 \%$ de las OG tienen esta antigüedad. La cantidad y antigüedad de estas permite inferir que los jóvenes son un asunto relativamente nuevo en la agenda pública; sobre todo en la del Estado.

Otra perspectiva importante para analizar a estas entidades es su capacidad organizacional en cuanto a la cantidad y tipo de personal que emplean, el alcance de la población que atienden, las instalaciones con las cuales cuentan, así como su presupuesto global y sus fuentes de financiamiento. Los datos de la encuesta muestran que ambos tipos de organizaciones tienen una capacidad precaria para hacer frente a la complejidad de los problemas sociales como al número de población atendida, esto resulta evidente en el poco personal de tiempo completo y en el bajo presupuesto anual (Tabla III). 
Tabla III

Capacidad organizacional entre OG y OSC en México

\begin{tabular}{|l|c|c|}
\hline \multicolumn{1}{|c|}{ Indicador } & OG & OSC \\
\hline Personal empleado tiempo completo (1 a 5 personas) & $43.2 \%$ & $56.5 \%$ \\
\hline Voluntarios (1 a 5 personas) & $36.1 \%$ & $32.6 \%$ \\
\hline Total del personal (11 a 20 personas) * & $17.6 \%$ & $26.4 \%$ \\
\hline Atención exclusiva a jóvenes de 12 a 29 años & $46.3 \%$ & $94.7 \%$ \\
\hline $\begin{array}{l}\text { Alcance de la población que atienden de 12 a 29 años } 11 \\
\text { a 100 personas) }\end{array}$ & $2.1 \%$ & $55.6 \%$ \\
\hline $\begin{array}{l}\text { Alcance de la población que atienden de 12 a 29 años } \\
\text { (más de 100,000 personas) }\end{array}$ & $51.6 \%$ & $0 \%$ \\
\hline Instalaciones físicas (propias, rentadas o prestadas) & $100 \%$ & $89.6 \%$ \\
\hline Sin instalaciones físicas (operan desde el ciberespacio) & $0 \%$ & $10.4 \%$ \\
\hline $\begin{array}{l}\text { Presupuesto anual durante el 2016 } \\
\text { (<1'000,000 MXN/ 51,894 USD) ** }\end{array}$ & $54.8 \%$ & $56.4 \%$ \\
\hline $\begin{array}{l}\text { Presupuesto anual durante el 2016 } \\
\text { (1'000,000-10'000,000 MXN/ 51,894-518,941 USD) } * *\end{array}$ & $29.4 \%$ & $30.9 \%$ \\
\hline $\begin{array}{l}\text { Presupuesto anual durante el 2016 } \\
\text { (> 10'000,000 MXN/ 518,941 USD)** }\end{array}$ & $15.7 \%$ & $12.6 \%$ \\
\hline
\end{tabular}

Fuente: elaboración propia a partir de 203 encuestas $^{5}$

El origen del presupuesto de las organizaciones es diversificado. En el caso de las OG, dado que la mayoría son municipales, la fuente presupuestal que sobresale es el municipio (43.4\%), seguido de las estatales (13.7\%) y al final la federación (2.7\%). Estas organizaciones también reciben, en menor porcentaje, recursos de cuotas, donaciones de empresas y particulares, empresas sociales, organismos internacionales, patronatos y generación de recursos propios. Mientras que la principal fuente de recursos de las OSC son el cobro de cuotas a la población beneficiaria (20.6\%), financiamiento federal (18.8\%) y empresas sociales $(10.2 \%)$, con menor participación, donaciones de empresas o particulares, financiamiento estatal, internacional, recursos propios y patronatos.

5. */ Integra a personal de tiempo completo, medio tiempo, honorarios y voluntarios. $* *$ Los pesos mexicanos MXN se convirtieron a dólares USD según conversión al 2016 de 1 MXN = 19.27 USD (http://www.eldolar. info/es-MX/mexico/dia/20161001). 
Los datos de la Tabla III, visibilizan capacidades similares entre las OG y las OSC, en cuanto a personal y presupuesto y ambas no cuentan con suficientes recursos humanos ni financieros para enfrentar los retos asociados a este sector creciente, con problemáticas diversas y complejas.

A pesar de algunas similitudes en la poca capacidad organizacional, habrá que destacar, como se advierte en la Tabla III, que un mayor número de OSC atiende exclusivamente a jóvenes con respecto a las OG. Aunque la mitad de las OG manifestaron una atención a más de 100 mil personas, esto se podría deber a que malinterpretaron la pregunta e incluyeron audiencias de campañas en medios de comunicación o TIC. Esto refuerza la tendencia que se ha identificado en el estudio de una mayor presencia especializada de las OSC en la atención al problema de los jóvenes frente al papel del Estado.

Otro dato que habrá que matizar es la existencia de organizaciones sin instalaciones físicas, esto se dio solo entre OSC, lo cual indica el hallazgo de una nueva modalidad de organizaciones que surgen y operan fundamentalmente desde el ciberespacio, estas son principalmente operadas por jóvenes con habilidades digitales.

Las formas de trabajo con los jóvenes que prevalecen son talleres, seminarios, cursos o clases, organización de eventos para ofrecer conferencias, charlas y sesiones informativas. 
Las principales áreas temáticas en las cuales se atienden a los jóvenes corresponden a una perspectiva tradicional de apoyo a los servicios de bienestar básicos. Es decir, al joven se le conceptualiza como una persona carente de formación que hay que proporcionarle educación en varios aspectos y actividades culturales y deportivas; o enmarcado en problemas como las adicciones, el embarazo temprano y la violencia, entre otros. Se identificaron muy pocas en las cuales se reconozca y promueva al joven como un ciudadano participativo y con voz propia capaz de generar cambios. En México la academia ha señalado que se requiere mayor atención de parte del gobierno hacia los jóvenes y cambiar la visión de tutelaje infantilizado que ha prevalecido. Además, señala que el Estado ha tendido a ejercer una política social con estrategias homogéneas ante los distintos grupos vulnerables, lo cual ha resultado en una invisibilización de la naturaleza específica de sus problemas (Corona, 2013).

\subsection{Infraestructura en TIC entre OG y OSC}

En ambos tipos de organizaciones existe una baja inversión en TIC (Tabla IV), poco menos de la mitad (42.2\%) de las organizaciones reporta cero inversiones. En el caso de las que sí invierten, las que dedican menos de 10,000 MXN/ 518 USD son mayoritariamente OSC (40\%), mientras que sólo el 15.5\% son OG. En el otro extremo, las que son capaces de invertir más de 75,000 MXN/ 3,892 USD solo se registra el $9.9 \%$ de las OG, sin presencia de las OSC. Por otra parte, más de la mitad de las organizaciones, $61.2 \%$ no cuenta con personal dedicado exclusivamente a tareas con respecto a las TIC, este porcentaje es aún mayor entre las OSC que las OG. 
Estos datos indican que en ambos casos no existe la posibilidad de aprovechar el potencial de las TIC dado que se invierte muy poco en infraestructura y personal especializado. No obstante, es evidente la mayor precariedad entre las OSC (Tabla IV), las cuales suelen no tener un presupuesto fijo y dependen de proyectos y financiamientos temporales. Esto genera un círculo vicioso donde no existe una estrategia que rompa las inercias y permita lograr mayores alcances de las organizaciones a partir del uso de TIC. Aunque se identificó un dato que sugiere que, a pesar de la deficiencia de recursos en ambos tipos de organizaciones, las OSC tiene una tendencia innovadora mayor. Las OSC adoptaron internet en 1992, mientras que las primeras OG tuvieron acceso en 1999.

Tabla IV

Infraestructura y acceso a TIC entre OG y OSC en México

\begin{tabular}{|l|c|c|}
\hline \multicolumn{1}{|c|}{ Indicador } & OG & OSC \\
\hline $\begin{array}{l}\text { Cuentan con más de 10 computadoras con acceso a } \\
\text { internet }\end{array}$ & $50.5 \%$ & $13.9 \%$ \\
\hline $\begin{array}{l}\text { Cuentan con más de 10 computadoras al servicio de la } \\
\text { población que atienden }\end{array}$ & $23.7 \%$ & $10.5 \%$ \\
\hline No invierten en infraestructura TIC & $42.3 \%$ & $42.2 \%$ \\
\hline $\begin{array}{l}\text { No cuentan con personal dedicado a tareas } \\
\text { exclusivamente relacionadas con TIC (Web master, } \\
\text { administrador de redes) }\end{array}$ & $49.4 \%$ & $71.7 \%$ \\
\hline
\end{tabular}

Fuente: elaboración propia a partir de 203 encuestas 


\section{1 \\ $\sum_{0}^{n}$}

4.3. Una propuesta para diagnosticar el aprovechamiento de TIC en organizaciones

La interpretación de los datos requirió contar con criterios claros para organizar los resultados y trascender lo descriptivo. Esto condujo a construir tres niveles posibles de uso de las TIC, de acuerdo al alcance y aprovechamiento: básico, intermedio y alto; a su vez, al interior de cada nivel se precisan dimensiones (Tabla V). Esto fue trabajado a partir de los datos recopilados del presente estudio y de los aportes de los diagnósticos de Finquelievich y Kisilevsky (2005) y Camacho (2003), así como las recomendaciones de la ONU (2010). Este marco analítico es una propuesta para realizar diagnósticos sobre el uso y aprovechamiento de las TIC entre organizaciones con fines de desarrollo social, además presenta horizontes ideales para un mejor uso de las TIC que pueden emplearse en el diseño de políticas públicas, programas de trabajo, cursos, talleres e intervenciones para la alfabetización digital a nivel organizacional.

Tabla V

Marco analítico. Aprovechamiento de las TIC para el fortalecimiento de OG y OSC

\begin{tabular}{|c|c|c|}
\hline Niveles & Dimensiones & Descripción según autores \\
\hline \multirow{2}{*}{$\begin{array}{l}\text { Básico } \\
\text { Uso interno y } \\
\text { cotidiano. El } \\
\text { aprovecha- } \\
\text { miento queda } \\
\text { confinado al } \\
\text { interior de la } \\
\text { organización. }\end{array}$} & $\begin{array}{l}\text { Apoyo para la } \\
\text { administración. }\end{array}$ & $\begin{array}{l}\text { El manejo y administración de los procesos de } \\
\text { información organizacional y transformación de los } \\
\text { procesos administrativos (Camacho, 2003). Monitorear } \\
\text { con precisión sus finanzas, ahorrar costos y operar de } \\
\text { manera efectiva al permitir a los empleados trabajar de } \\
\text { manera remota y flexible; y, mantener con seguridad } \\
\text { los datos de sus contactos (ONU, 2010). }\end{array}$ \\
\hline & $\begin{array}{l}\text { Facilitar los } \\
\text { flujos de } \\
\text { información y } \\
\text { comunicación } \\
\text { interna. }\end{array}$ & $\begin{array}{l}\text { En la gestión organizacional, la comunicación e } \\
\text { información interna, el uso del e-mail, el acercamiento } \\
\text { de sedes y personas (Camacho, 2003). Gestión eficiente } \\
\text { de la información (ONU, 2010). }\end{array}$ \\
\hline
\end{tabular}




\section{Intermedio}

Al uso

interno se

añade el uso

externo para

posicionarse

y brindar su

atención.

\section{Alto}

Se entiende a las TIC como herramientas para lograr el desarrollo y el cambio social.

\section{Difusión de la imagen y los servicios de la organización.}

\section{Búsqueda} de recursos humanos, financieros, materiales y de información.

Ampliación del alcance y calidad de su atención.

\section{Incrementar la} habilitación de sus integrantes.

Generación de conocimiento.

Promoción de la igualdad de oportunidades.

Responsabilidad Social.
Incrementar su perfil y visibilidad; así como proporcionar una forma eficiente de canalizar información sobre sus actividades y comprometerse con los grupos interesados, incluidos los donantes, el gobierno, el sector privado, el público en general y los socios de la comunidad (ONU, 2010).

Conectarse con una amplia gama de donantes individuales e institucionales, recaudar dinero y encontrar voluntarios, tanto a nivel local como internacional (ONU, 2010).

Comprender a quiénes emplean sus servicios y cómo pueden ampliar su alcance; así como permitir que los usuarios del servicio se apoyen entre sí a través de comunidades en línea (ONU, 2010). Recepción de quejas y sugerencias y promover la interacción con sus beneficiarios (aportación a partir de este diagnóstico). Los procesos de capacitación y e-learning, el intercambio y sistematización de experiencias (Camacho, 2003). Mantenerse al tanto de los desarrollos actuales y la legislación en su campo (ONU, 2010). Generar reflexividad y una comprensión amplia sobre la problemática social que atienden (aportación a partir de este diagnóstico).

La generación de conocimientos a través de estudios propios, la producción de contenidos para la red y la edición de sitios Web, la creación y difusión de bases de datos, bibliotecas virtuales, centros de documentación (Camacho, 2003).

La incorporación e impulso de las TIC para la igualdad de oportunidades, el uso con sentido y la apropiación social; impulso al desarrollo de la economía social, el desarrollo local y la promoción del empleo; así como promoción de la participación ciudadana, igualdad de acceso y satisfacción de derechos y fortalecimiento de las organizaciones comunitarias (Finquelievich \& Kisilevsky, 2005).

Transparencia y neutralidad tecnológica (aportación a partir de este diagnóstico). 


\begin{tabular}{|l|l|l|}
\hline $\begin{array}{l}\text { Articulación con } \\
\text { redes nacionales } \\
\text { e internaciona- } \\
\text { les para alcanzar } \\
\text { sus metas. }\end{array}$ & $\begin{array}{l}\text { El desarrollo de interrelaciones, la posibilidad de } \\
\text { mayor intercambio, la participación y conformación de } \\
\text { redes y el acceso a los movimientos globales (Camacho, } \\
\text { 2003). }\end{array}$ \\
\cline { 2 - 3 } $\begin{array}{l}\text { Inserción de las } \\
\text { problemáticas } \\
\text { locales en } \\
\text { las agendas } \\
\text { nacionales y } \\
\text { globales. }\end{array}$ & $\begin{array}{l}\text { La difusión de posturas, posicionamientos y } \\
\text { promoción de campañas temáticas (Camacho, 2003). } \\
\text { Participación, cogestión y evaluación de políticas } \\
\text { públicas para su mejora; así como la articulación desde } \\
\text { lo local a lo global para construir ciudadanía mundial } \\
\text { (Finquelievich \& Kisilevsky, 2005). }\end{array}$ \\
\hline
\end{tabular}

Fuente: elaboración propia a partir de 203 encuestas y revisión de autores (Camacho; 2003; Finquelievich \& Kisilevsky, 2005; ONU, 2010)

A partir de esta tabla se ofrecen los resultados globales presentados en ambas organizaciones, presentando en cada nivel los usos en el orden del mayor porcentaje identificado al menor y comentando los casos en los cuales destacan algunas diferencias significativas entre los dos tipos de organizaciones. En la encuesta las opciones de respuesta fueron: de manera muy frecuente (diariamente), algo frecuente (semanalmente), poco frecuente (menos que mensualmente) o nunca. Los porcentajes que aquí aparecen corresponden al resultado global de ambos tipos de organizaciones, OG y OSC sumando los resultados de las respuestas muy frecuente y algo frecuente. 


\subsection{Nivel básico}

Este nivel se refiere a la relación elemental que establecen las organizaciones con las TIC. Se trata de un uso interno y cotidiano donde el aprovechamiento queda confinado a facilitar los procesos administrativos y los flujos de información y comunicación interna. Las dimensiones del nivel son:

a) Apoyo para la administración. Las organizaciones estudiadas aprovechan las TIC para: calendarizar y programar actividades (65.0\%); almacenar en la nube (58.1\%); investigar precios y cotizaciones (56.1\%); sistematizar la contabilidad a través del uso de software especializado (43.8\%); manejar cuentas bancarias por internet (43.3\%); gestionar proyectos mediante software especializado (35.4\%); pagar a proveedores por banca de internet (35.0\%); usar bases de datos y plataformas internas en línea para el seguimiento de procesos $(31.1 \%)$ y pagar nómina de empleados (28.1\%).

b) Facilitar los flujos de información y comunicación interna. De manera muy frecuente $\mathrm{y}$ algo frecuente, las organizaciones dan avisos o se comunican internamente con el personal mediante el uso de TIC como Facebook, Whatsapp, Messenger, correo electrónico, intranet y Skype (84.2\%). Este nivel prevalece en las organizaciones estudiadas.

\subsubsection{Nivel intermedio}

Este nivel, a diferencia del anterior, alude a las actividades de la organización que desbordan sus procesos internos y la pone en contacto con el exterior en tres dimensiones. La primera es la más común que se refiere a los usos de las TIC para dar a conocer la imagen de la organización. En segundo lugar, se emplean las TIC para buscar recursos, sean financieros, materiales, de información o humanos como el reclutamiento de voluntarios. En una tercera dimensión, existe la posibilidad del empleo de TIC para ampliar el alcance de su atención. 
a) Difusión de la imagen y los servicios de la organización. Prevalece el empleo de redes sociales para difundir su imagen, principalmente Facebook (91.6\%), le sigue Twitter (51.7\%) y en tercer lugar Youtube (29.1\%). Además, 74.4\% de las organizaciones distribuyen sus campañas de comunicación estratégica a través de TIC. Estas son las dimensiones identificadas en este nivel.

b) Búsqueda de recursos humanos, financieros, materiales y de información. De manera frecuente y muy frecuente, las organizaciones buscan información en sitios relacionados con su actividad (el 90.6\%); en bases de datos y estadísticas $(77.3 \%)$ y en informes, reportes, textos académicos y literatura especializada (76.4\%). Sin embargo, el 70\% no usa las TIC para buscar información estratégica que les permita acceder a fuentes alternativas de recursos humanos, financieros o de divulgación de su misión social. En el caso de emplear TIC para buscar recursos, el $66.5 \%$ realizan campañas para captar voluntarios y el $29.6 \%$ las emplean para buscar donadores.

c) Ampliación del alcance y calidad de su atención. En esta dimensión sobresale (88.7\%) el uso de TIC para dar a conocer los servicios que ofrecen. El 69.5\% brinda a través de ellas consultorías y capacitación, el 61.6\% recibe quejas y sugerencias en soportes digitales y el $61.1 \%$ organiza sus actividades en calendarios, mientras que un $4.6 \%$ ofrece videos tutoriales. Por otra parte, la población objetivo interactúa con las organizaciones predominantemente a través de las siguientes modalidades: plantean comentarios en las redes sociales $(88.7 \%)$, la población que atienden sube fotos y vídeos (77.3\%), y producciones originales (48.8\%). En un menor grado, las organizaciones distribuyen encuestas y formularios para que se respondan en línea (28.1\%) y menos aún para evaluar su desempeño (20.2\%).

En este nivel hay un vacío de usos que se relacionen con la búsqueda de información para proyectos o financiación alternativos, tampoco para interactuar de manera más sistemática y eficiente con los jóvenes que son la población que atienden. 


\subsubsection{Nivel alto}

Este estrato implica una visión y prácticas más complejas de las TIC; sobre todo el comprender que tienen un papel participativo en la resolución de los problemas locales y que permiten insertarlos en la agenda global de desarrollo y cambio social. Las dimensiones de este nivel son:

a) Incrementar la habilitación de sus integrantes. A pesar de que las TIC representan una amplia gama para capacitar a los miembros de las organizaciones, menos de la mitad, el 48.8\% las emplean para esta función.

b) Generación de conocimiento. Esta dimensión se refiere a indagar y ampliar el conocimiento sobre la misión social que atienden, menos de la mitad (46.3\%) producen recursos o materiales propios en línea (vídeos, presentaciones, infografías, podcast, entre otros); enseguida generan y distribuyen publicaciones digitales (25.1\%); otras ofrecen centros de documentación (18.2\%) y bibliotecas virtuales $(9.4 \%)$.

c) Promoción de la igualdad de oportunidades. No se identificaron datos que se pudieran insertar en esta posibilidad.

d) Responsabilidad Social. Las organizaciones de manera poco frecuente o nunca rinden cuentas por internet a través de una página propia o de otra institución (59.6\%). Incluso, el 52.2\% no respondió sobre cuál fue la suma de su presupuesto durante el año anterior (2015); la encuesta se aplicó a finales del 2016. Es importante aclarar que a las organizaciones se les solicitó este dato solo vía encuesta y no formalmente a través del Instituto Nacional de Transparencia, Acceso a la Información y Protección de Datos Personales (INAI, 2018) que es el instituto regulador de transparencia en cuanto al gasto de fondos públicos en el país. 
e) Articulación con redes nacionales e internacionales para alcanzar sus metas. Un poco menos del conjunto global $(48.8 \%)$ de las organizaciones pertenecen a una red de asociaciones o instituciones. Cabe destacar que son las OSC las que más lo hacen (54.6\%) frente a las OG (42.1\%). Los alcances de estas redes, son principalmente a nivel nacional (40\%), menos en el estatal $(35 \%)$ y aún más reducido en el internacional (5\%). Esto sugiere que el trabajo social realizado en el país está poco integrado en la comunidad internacional y como resultado carece del apoyo que esto podría significar.

f) Inserción de las problemáticas locales en las agendas nacionales y globales. Se identificaron redes de apoyo para impulsar políticas públicas y/o legislaciones (44.8\%); denuncias (21.7\%); apoyo a causas (21.2\%) y movilizaciones sociales $(18.2 \%)$.

En México, continúa el crecimiento de la penetración de internet (63\%, según la Asociación de Internet.mx, 2018), lo cual no significa que se haga un uso adecuado de él para el desarrollo humano y social. Esto se puede comprobar con los anteriores datos sobre el retraso en las organizaciones en cuanto a la inversión en infraestructura y personal especializado en TIC.

El aprovechamiento de las TIC descrito en este nivel no es óptimo, un poco menos de la mitad aproximadamente de las organizaciones las emplean para capacitar a su personal, para generar conocimiento o para integrarse en redes nacionales. Los porcentajes más bajos se encuentran en actividades de investigación, en la participación y colaboración en redes internacionales y en materia de responsabilidad social, en particular en lo que se refiere a la transparencia en el manejo de sus financiamientos públicos. En el título donde no se encontró ninguna actividad reportada fue en promover la igualdad de oportunidades. 


\section{Discusión y conclusiones}

Este artículo busca definir con mayor claridad la agenda de estudio de la línea de TIC para el desarrollo (ICT4D), al precisar el nivel organizacional como un campo concreto de investigación e intervención para fortalecer, a través de las TIC, la capacidad de las organizaciones que tienen como propósito una causa social. A partir del diagnóstico de los usos y aprovechamiento de las TIC entre OG y OSC para el desarrollo social en México, se propone una clasificación de niveles y dimensiones que permitirá analizar otros casos de organizaciones, problemáticas y sectores de la población.

La clasificación facilita la comprensión de las posibilidades de aprovechamiento de las TIC para realizar diagnósticos, evaluaciones, planes de trabajo y principalmente plantea un horizonte para ampliar la visión de las organizaciones. En el campo de las políticas públicas y los programas de alfabetización digital, contribuye agenerar estrategias distintas a las centradas en apoyar a comunidades a través de las TIC. Se propone, además de continuar con el trabajo entre los sectores más vulnerables, la necesidad de reconocer a las organizaciones con fines sociales como actores clave para aprovechar las lógicas de la sociedad del conocimiento con el fin de atender problemas sociales y fortalecer su capacidad organizacional. En este sentido, se identificaron escasos trabajos, y de manera más específica encuestas que realizarán un diagnóstico sobre TIC entre organizaciones con propósitos sociales. Esta tarea se apunta como parte de una agenda de futuros estudios en otros contextos, no sólo para realizar diagnósticos en un momento dado, sino además de manera longitudinal para valorar si existe un posible progreso en el uso de TIC en el campo de las organizaciones para el desarrollo social. La clasificación de niveles y dimensiones de aprovechamiento de las TIC representa una guía para generar con mayor precisión líneas de acción y la habilitación de las organizaciones. 
En el caso de México, se puede destacar que, a pesar de la baja infraestructura y personal especializado en TIC, estas se emplean en los niveles básicos e intermedios. Sin embargo, falta mayor visión y capacitación entre estas organizaciones para comprender el papel, no meramente de las TIC, sino de la sociedad de la información y el conocimiento para enfrentar la exclusión social. Lo que acontece en estas organizaciones coincide con el nivel de aprovechamiento de las TIC entre la población en general. Lamentablemente, a pesar del crecimiento acelerado de la penetración de internet y la reforma constitucional en materia de telecomunicaciones en 2013, que plantea como un derecho de los ciudadanos la integración en la sociedad de la información y comunicación para la democracia y la participación ciudadana (Ramos, 2015), esto no acontece. Actualmente en el país, como en el mundo, se discute la distancia entre el potencial y el papel real de las TIC, principalmente el de las redes sociales para contribuir a estos ideales.

\section{Agradecimientos}

Este proyecto fue financiado por el Fondo Sectorial de Investigación para la Educación, SEP/CONACYT y la Universidad Autónoma de Aguascalientes, México. Además, un agradecimiento al Dr. Renato González Sánchez por su apoyo en la definición de la muestra y el esquema de confianza del instrumento. 


\section{Bibliografía}

- ASOCIACIÓN DE INTERNET.MX/INFOTEC. (mayo de 2018). Estudio sobre los Hábitos de los Usuarios de Internet en México 2017 (13º). Recuperado de https://www.infotec.mx/work/models/infotec/Resource/1012/6/images/ Estudio_Habitos_Usuarios_2017.pdf

- ATTOUNI, M. A. K., \& MUSTAFFA, C. S. (2014). How do non-profit organizations in Libya adopt and use social media to communicate with the society? ProcediaSocial and Behavioral Sciences, (155), 92-97.doi: 10.1016/j.sbspro.2014.10.262

- CAMACHO, K. (2003). Internet, ¿cómo vamos cambiando?. San José de Costa Rica: Fundación Acceso.

- CATALANO, R. F., BERGLUND, M. L., RYAN, J. A., LONCZAK, H. S., \& HAWKINS, J. D. (2004). Positive youth development in the United States: Research findings on evaluations of positive youth development programs. The Annals of the American Academy of Political and Social Science, 591(1), 98-124. doi: $10.1177 / 0002716203260102$

- Corona, S. (2013, 3, abril). Críticas en México a la política de Peña Nieto hacia los jóvenes. El País. Recuperado de https://elpais.com/ internacional/2013/04/03/actualidad/1364955683_507552.html

- DE SILVA, N. (2016). Exploring the use of ICTs in non-profit sector organisations: supporting the third act (Tesis doctoral). University of Wellington, Nueva Zelanda. Recuperado de https://researcharchive.vuw.ac.nz/xmlui/bitstream/ handle $/ 10063 / 5297 /$ thesis.pdf?sequence=1 
- DURAND, B. (2017). Ninis: factores determinantes. Realidad, datos y espacio. Revista Internacional de Estadística y Geografía, 8(3), 46-72.

- EL COLEGIO DE MÉXICO. (2018). Red de Estudios sobre Desigualdades. Desigualdades en México/2018. Recuperado de https://desigualdades.colmex. $\mathrm{mx}$ /informe-desigualdades-2018.pdf

- ENZ, A., FRANCO, V. \& SPAGNUOLO, V. (2012). Manual de comunicación para organizaciones sociales: hacia una gestión estratégica y participativa. Argentina: Comunia.

- FINQUELIEVICH, S., \& KISILEVSKY, G. (2005). La sociedad civil en la era digital: Organizaciones comunitarias y redes sociales sustentadas por TIC en Argentina (Documento de trabajo no 41). Argentina: Universidad de Buenos Aires. Recuperado de http://lanic.utexas.edu/project/laoap/iigg/dt41.pdf

- INSTITUTO NACIONAL DE ESTADÍSTICA E INFORMÁTICA (INEI). (2007). Encuesta sobre Tecnologías de la Información y Comunicaciones. Recuperado de http://www.cepal.org/socinfo/noticias/paginas/6/35876/PeruEMPRESAS. pdf

- INSTITUTO NACIONAL DE ESTADÍSTICA Y GEOGRAFÍA (INEGI). (2017). Estadísticas sobre disponibilidad y uso de tecnología de información y comunicaciones en los hogares, ENDUTIH. México: INEGI. Recuperado de http://www.beta.inegi.org.mx/programas/dutih/2017/ 
- INSTITUTO NACIONAL DE TRANSPARENCIA, ACCESO A LA INFORMACIÓN Y PROTECCIÓN DE DATOS PERSONALES (INAI). (2018). ¿Qué es el INAI? . Recuperado de: http://inicio.inai.org.mx/SitePages/que-es-el-inai.aspx

- KLEINE, D. (2013). Technologies of choice: ICTs, development and capabilities approach. Estados Unidos: MIT Press.

- LIZARRAGA, G. (2004). Organizaciones civiles y gobierno: un eslabón estratégico en la transición. Estudios Fronterizos, 5(10), 51-72.

- MONTESINOS-LÓPEZ, O. A., LUNA-ESPINOZA, I., HERNÁNDEZ-SUÁREZ, C. M. \& TINOCO-ZERMEÑO, M. A. (2019). Muestreo estadístico. Tamaño de muestra y estimación de parámetros. Universidad de Colima: México.

- NEGRETE, R. \& LEYVA, G. (2013). Los Ninis en México: una aproximación crítica a su medición. Realidad, datos y espacio. Revista Internacional de Estadística y Geografía, 4(1), 90-121.

- NUGROHO, Y. (2011). Opening the black box: The adoption of innovations in the voluntary sector-The case of Indonesian civil society organizations. Research Policy, 40(5), 761-777. doi: 10.1016/j.respol.2011.03.002

- ORGANIZACIÓN DE LAS NACIONES UNIDAS (ONU). (2010). Why are ICTS important for Civil Society Organizations?. Recuperado de: http://www.un.org/ esa/socdev/ngo/docs/2010/directory/ictcso.pdf 
- ORGANIZACIÓN PARA LA COOPERACIÓN Y DESARROLLO ECONÓMICOS (OCDE). (2018). Getting it Right: Prioridades estratégicas para México. Recuperado de http//dx.doi.org/10.1787/9789264292871-es

- PALOS, M. \& MÉNDEZ, E. (2011). Las organizaciones sin fines de lucro en México. Un análisis crítico sobre los actuales efectos de su gestión. En M. Cano \& J. Ortiz. (Coords.), Contabilidad, autoría y fiscal. Mesa llevada a cabo en el Congreso Internacional de Investigación en Ciencias Administrativas. Veracruz: México.

- RAMOS,H. (2015). Información y ciudadanía, una propuesta desde la gobernanza. Investigación Bibliotecológica: Archivonomía, Bibliotecología e Información, 29(67), 113-140. doi: https://doi.org/10.1016/j.ibbai.2016.02.039

- REGUILlO, R. (2012). Culturas juveniles. Formas políticas del desencanto. Buenos Aires: Siglo Veintiuno.

- REYGADAS, R. (2005). Aportes de la sociedad civil al desarrollo local en México. Revista Futuros, 3(11), 1-9. Recuperado de http://www.asocam.org/sites/ default/files/publicaciones/files/4fc8d4751ef6284a77c3bab86f8427d6.pdf

- SEN, A. (2000). Desarrollo y libertad. Barcelona: Planeta.

- TUFTE, T. (2015). Comunicación para el cambio social. La participación y el empoderamiento como base para el desarrollo mundial. Barcelona: Icaria.

- WALSHAM, G. (2017). ICT4D research: Reflections on history and future agenda. Information Technology for Development, 23(1), 18-41. doi: https://doi.org/10. 1080/02681102.2016.1246406

- WORLD SUMMIT ON THE INFORMATION SOCIETY (WSIS). (2018). Recuperado de https://www.itu.int/net4/wsis/forum/2018/ 


\title{
Biografías
}

\section{María Rebeca Padilla de la Torre}

Universidad Autónoma de Aguascalientes, México

http://orcid.org/0000-0002-5881-3958

rebecapadilla.uaa@gmail.com,rebeca.padilla@edu.uaa.mx

Profesora-investigadora del Departamento de Comunicación de la Universidad Autónoma de Aguascalientes, México. Doctora en Estudios Científico-Sociales en la especialidad de Comunicación, Cultura y Sociedad por el Instituto Tecnológico de Estudios Superiores de Occidente. Estudia las líneas de investigación del análisis sociocultural y político de las prácticas mediáticas y las tecnologías de información y la comunicación para el desarrollo. Líder del Cuerpo Académico de Estudios Socioculturales y Miembro del Sistema Nacional de Investigadores nivel 2.

\author{
Ana Isabel Zermeño Flores \\ Universidad de Colima, México \\ http://orcid.org/0000-0001-7371-8767 \\ anaz@uco.mx
}

Profesora-Investigadora del Centro Universitario de Investigaciones Sociales de la Universidad de Colima, México. Fundadoray coordinadora de "Agorante", Grupo de Investigación en Sociedad y Tecnologías. Doctora en Comunicación Audiovisual. Líneas de investigación: TIC para el desarrollo y el cambio social; evaluación e impacto social de las tecnologías; inclusión digital e intervención comunitaria; vulnerabilidad digital; estudios de cultura contemporánea; juventud; usabilidad web; gestión de la información. 


\section{Thomas Tufte}

Loughborough University, London, United Kingdom

http://orcid.org/0000-0003-3253-8481

T.Tufte@lboro.ac.uk

Profesor Titular y Director del Institute of Media and Creative Industries; en Loughborough University London, Reino Unido (2018actualidad). Es también "Senior Research Associate" en la Universidad de Johannesburgo (Sudáfrica, 2013-actualidad). Es Doctor en Comunicación por la Universidad de Copenhague, Dinamarca (1995). Sus intereses de investigación se orientan hacia el papel de la comunicación en la articulación y movilización de ciudadanos en procesos de cambio. Sus proyectos de investigación se han enfocado en procesos de cambio en África y América Latina. Además de investigador, ha realizado numerosas consultorías en el campo de comunicación y cambio social para organizaciones internacionales como agencias de las Naciones Unidas (UNICEF, PNUD, UNESCO), fundaciones y ONGs internacionales (como Fundación Rockefeller, Panos y ADRA) y organizaciones bilaterales (USAID, Danida y SIDA). 